Article

\title{
Angular Regioselectivity in the Reactions of 2-Thioxopyrimidin-4-ones and Hydrazonoyl Chlorides: Synthesis of Novel Stereoisomeric Octahydro[1,2,4]triazolo[4,3-a]quinazolin-5-ones
}

\author{
Awad I. Said ${ }^{1,2}$, , Márta Palkó $^{1,3, *(\mathbb{D}}$, Matti Haukka ${ }^{4}$ and Ferenc Fülöp ${ }^{1,3}$ \\ 1 Institute of Pharmaceutical Chemistry, University of Szeged, Eötvös u. 6, H-6720 Szeged, Hungary; \\ awadsaid@aun.edu.eg (A.I.S.); fulop@pharm.u-szeged.hu (F.F.) \\ 2 Chemistry Department, Faculty of Science, Assiut University, Assiut 71516, Egypt \\ 3 Interdisciplinary Excellence Center, Institute of Pharmaceutical Chemistry, University of Szeged, \\ H-6720 Szeged, Hungary \\ 4 Department of Chemistry, University of Jyväskulä, FIN-40014 Jyväskulä, Finland; matti.o.haukka@jyu.fi \\ * Correspondence: palko.marta@szte.hu; Tel.: +36-62-341966; Fax: +36-62-545705
}

Received: 4 November 2020; Accepted: 27 November 2020; Published: 1 December 2020

check for updates

\begin{abstract}
The regioselective synthesis of cis and trans stereoisomers of variously functionalized octahydro[1,2,4]triazolo[4,3-a]quinazolin-5-ones was performed. The 2-thioxopyrimidin-4-ones used in the synthesis reacted with hydrazonoyl chlorides in a regioselective manner to produce the angular regioisomers [1,2,4]triazolo[4,3-a]quinazolin-5-ones rather than the linear isomers [1,2,4]triazolo[4,3-a] quinazolin-5-ones. The synthesis process took place with electronic control. The angular regiochemistry of the products was confirmed by X-ray experiments and two-dimensional NMR studies.
\end{abstract}

Keywords: regioselective reactions; hydrazonoyl chlorides; 2-thioxopyrimidin-4-ones; [1,2,4]triazolo $[4,3-a]$ quinazolin-5-ones

\section{Introduction}

The $[1,2,4]$ triazolo[4,3-a]pyrimidinone scaffold has been known to exhibit a wide range of pharmacological activities such as antitumor, anti-inflammatory, antimicrobial, and antifungal activity, as well as macrophage activation [1-9].

A reaction between hydrazonoyl chlorides decorated with different functionalities [10-12] and 2-thioxopyrimidin-4-ones is an efficient strategy for incorporating the $[1,2,4]$ triazolo moiety into $[1,2,4]$ triazolo[4,3-a]pyrimidinones $[13,14]$.

Recently, we reported that 2-thioxopyrimidin-4-one constructed on the norbornene skeleton gave an angular regioisomer ([1,2,4]triazolo[4,3-a]pyrimidin-7(1H)-one), functionalized with various hydrazonoyl chlorides, as the sole product of the reaction [15]. This was in contrast to findings observed previously, where $[1,2,4]$ triazolo[4,3-a]pyrimidin-5(1H)-one, the linear regioisomer, was the sole product of the reaction [16-21].

Herein, we report the extension of our research for the regioselective synthesis of novel cis- and trans-octahydro[1,2,4]triazolo[4,3-a]quinazolin-5-ones $\mathbf{4 a - g}$ and $\mathbf{5 a - g}$ via the reaction of cyclohexane-fused cis- or trans-2-thioxopyrimidin-4-ones $\mathbf{1}$ and $\mathbf{2}$ with hydrazonoyl chlorides $\mathbf{3 a}-\mathbf{g}$, taking place under electronic control. Moreover, X-ray and two-dimensional NMR studies were used to prove the stereochemistry of the products. 


\section{Results and Discussion}

Cyclohexane-fused cis- and trans-2-thioxopyrimidin-4-one $\mathbf{1}$ and $\mathbf{2}$ were prepared according to previously described procedures [22]. The thioxopyrimidinone derivatives 1 or $\mathbf{2}$ thus prepared were reacted with the hydrazonoyl chlorides $3 \mathbf{a}-\mathbf{g}$ bearing varied functionalities in dioxane in the presence of triethylamine as a base under reflux conditions (Scheme 1). According to the reaction mechanism depicted in Scheme 2, the angular regioisomers [1,2,4]triazolo[4,3-a]quinazolin-5(3H)-one 4a-g and $5 \mathrm{a}-\mathrm{g}$ and linear regioisomers $[1,2,4]$ triazolo[4,3-a]quinazolin-5(3H)-one $\mathbf{6} \mathbf{a}-\mathbf{g}$ and $\mathbf{7} \mathbf{a}-\mathbf{g}$ were expected to be formed. The outcome of the reactions depends on the involvement of the tautomeric structures I or II of the cyclohexane-fused 2-thioxopyrimidin-4-ones $\mathbf{1}$ and $\mathbf{2}$. The reactions proceeded through S-alkylation [17-21] to give S-alkylated products A followed by Smiles rearrangement [23], affording intermediates $\mathbf{B}$, which cyclized in situ under the employed reaction conditions via the elimination of hydrogen sulfide gas to give the desired products $4 \mathbf{a}-\mathbf{g}$ and $\mathbf{5 a}-\mathbf{g}$ [20]. As evidenced by TLC and NMR spectroscopy, the transformations took place in a regioselective manner, producing the corresponding angular regioisomers as the sole products.

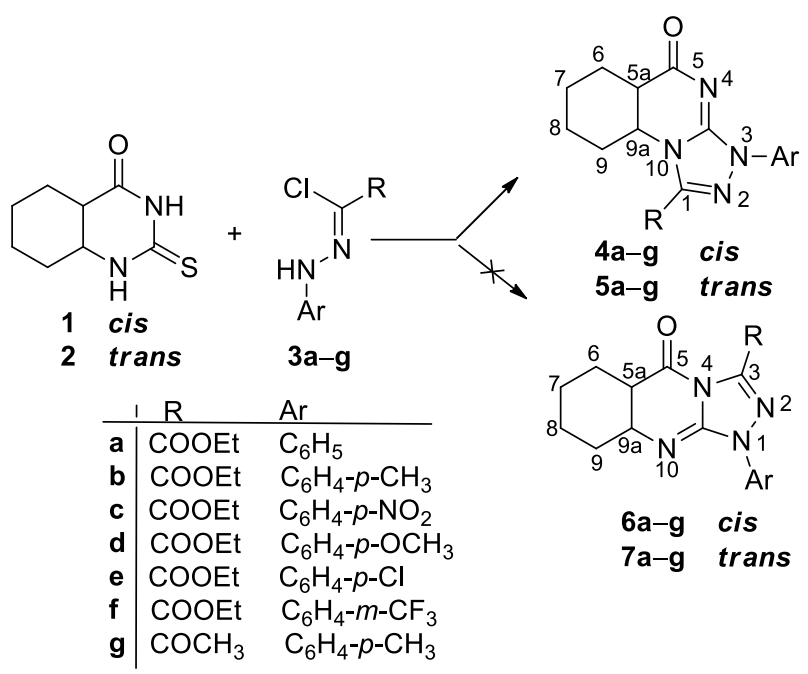

Scheme 1. Synthesis of $[1,2,4]$ triazolo[4,3-a]quinazolin-5(3H)-one $4 \mathbf{a}-\mathbf{g}$ and $\mathbf{5 a - g}$.

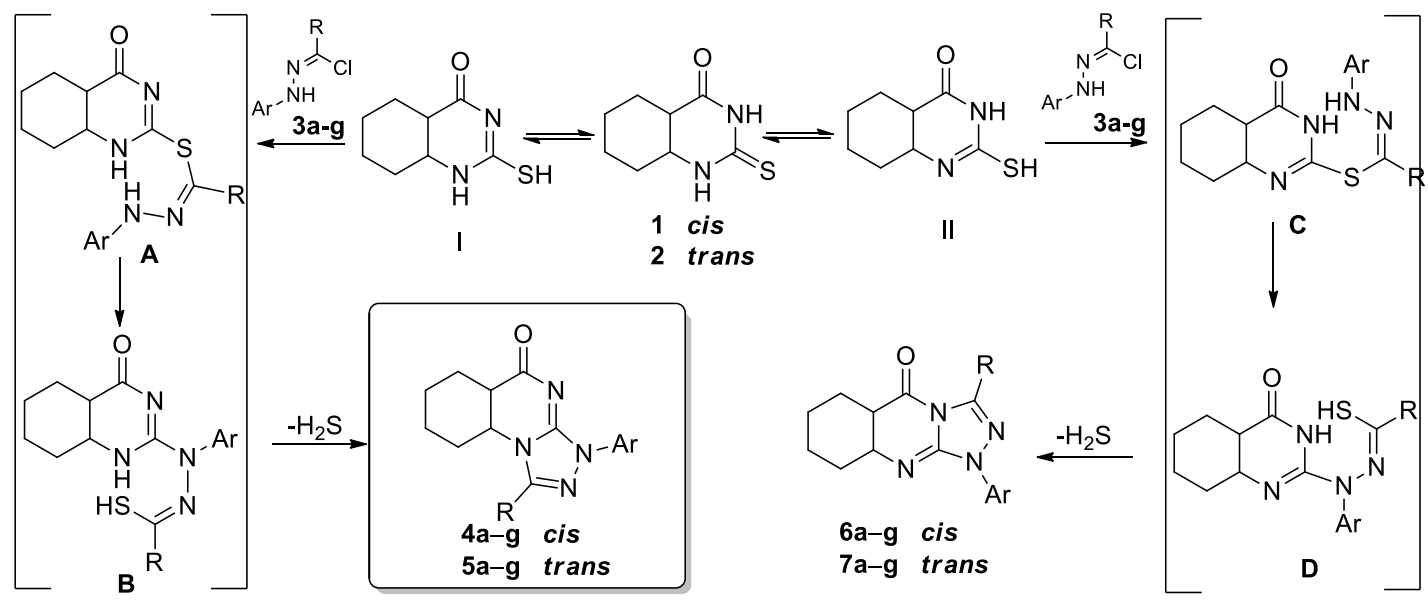

Scheme 2. Proposed reaction pathways to form angular and linear regioisomers.

The steric structure of the angular regioisomers was evidenced with information acquired through various instrumental techniques, namely, ${ }^{1} \mathrm{H}-\mathrm{NMR},{ }^{13} \mathrm{C}-\mathrm{NMR}$, and two-dimensional NMR including NOESY (neighboring Overhauser effect spectroscopy correlation), HMBC (heteronuclear multiple 
bond correlation), and X-ray crystallographic analysis. The ${ }^{1} \mathrm{H}-\mathrm{NMR}$ spectra of the products formed by the hydrazonoyl chloride ethyl esters $3 a-f$ show a more multiplicated signal pattern corresponding to the $\mathrm{CH}_{2}$ moiety of the ester functional group (Supplementary Materials), which suggests the steric proximity of the ester group and the cyclohexane skeleton. Moreover, the NOESY spectra exhibit a mutual correlation between the hydrogens of $\mathrm{CH}_{2}$ and cyclohexane. In addition, the HMBC spectra show a mutual correlation between $\mathrm{H}-9 \mathrm{a}$ and $\mathrm{C}-1$, which are separated by three bonds in the angular regioisomers. However, this correlation cannot exist in the linear regioisomers, because the $\mathrm{C}-3$ and $\mathrm{H}-9 \mathrm{a}$ atoms are separated by five bonds (Figure 1a). Last but not least, the ${ }^{13} \mathrm{C}-\mathrm{NMR}$ spectra reveal the signal of the carbonyl carbon of the pyrimidinone ring residue at nearly $176 \mathrm{ppm}$. These chemical shift values are similar to those of annelated pyrimidinones of type A rather than those of type $\mathbf{B}$ (Figure 1b) [24]. Finally, the X-ray crystallographic analysis of $\mathbf{5} \mathbf{b}$ provided conclusive evidence for the angular regiochemistry of the products (Figure 2).

angular regioisomer

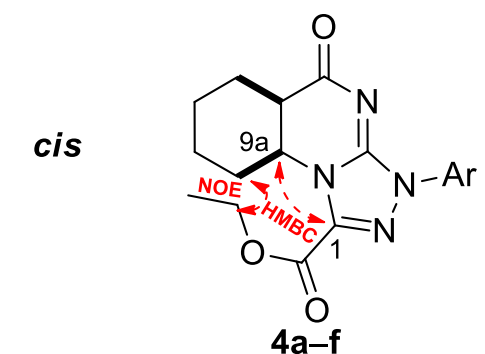

trans

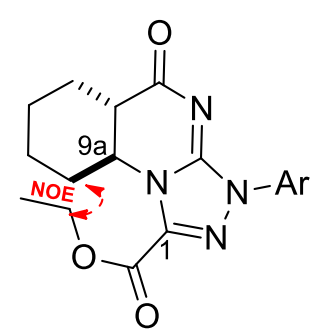

$5 a-f$

\section{linear regioisomer}<smiles>CCOC(=O)C1=NN([Al])C2=N[C@@H]3CCCC[C@H]3C(=O)N12</smiles>

$6 a-f$

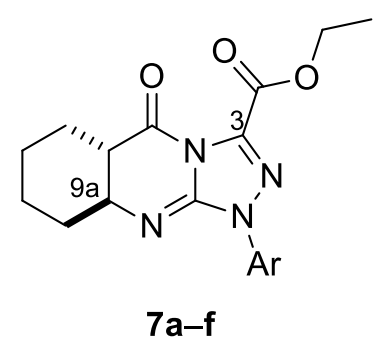

(a)

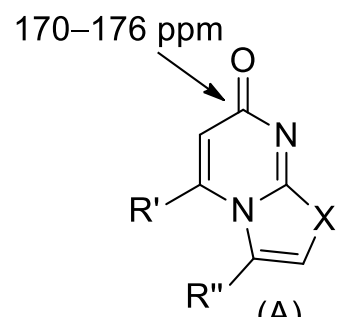

(A)

161-164 ppm<smiles>[X]c1cc([R])n2c(=O)cc([R])nc2n1</smiles>

(B)

Figure 1. (a) Heteronuclear multiple bond correlation (HMBC) and neighboring Overhauser effect (NOE) mutual correlations in angular regioisomers, and the lack of a similar correlation in their linear counterparts. (b) ${ }^{13} \mathrm{C}-\mathrm{NMR}$ data used for assigning the stereochemistry of the products.

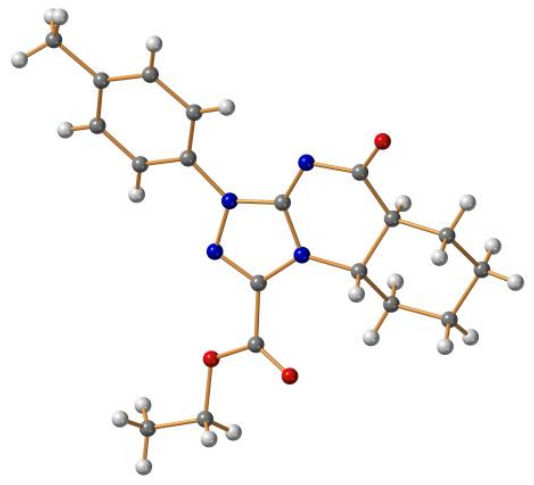

Figure 2. TELP image of $5 \mathbf{b}$ at $50 \%$ probability level.

On the basis of the above evidence, the angular structures $4 \mathbf{a}-\mathbf{g}$ and $\mathbf{5 a}-\mathbf{g}$ were assigned for the products, and, consequently, the linear structures $6 \mathbf{a}-\mathbf{g}$ and $7 \mathbf{a}-\mathbf{g}$ could be rejected. 
The regioselectivity of these reactions delivering the angular regioisomers was ascribed to electronic factors rather than steric factors. That is, since the tautomeric form I is electronically and energetically predominant, the reaction proceeds through tautomeric form I and leads to the formation of the angular regioisomer (Scheme 2).

\section{Materials and Methods}

\subsection{General Methods}

NMR analyses were performed at 500.20 MHz for ${ }^{1} \mathrm{H}-\mathrm{NMR}$ and at $125.62 \mathrm{MHz}$ for ${ }^{13} \mathrm{C}-\mathrm{NMR}$ in $\mathrm{CDCl}_{3}$ at room temperature, using a Bruker AV NEO Ascend 500 spectrometer (Bruker Biospin, Karlsruhe, Germany) with a Double Resonance Broad Band Probe (BBO). Tetramethylsilane (TMS) was used as an internal standard. The reactions were monitored by thin-layer chromatography (TLC) using aluminum sheets coated with silica gel (POLYGRAM ${ }^{\circledR}$ SIL G/UV254, Merck, Kenilworth, NJ, USA). The TLC plates were visualized under UV light. The melting points were measured using a Hinotek-X4 micro melting point apparatus (Hinotek, Ningbo, China).

The cyclohexane-fused cis- and trans-2-thioxopyrimidin-4-ones $\mathbf{1}$ and $\mathbf{2}$ were prepared from the corresponding amino esters according to reported procedures [25-27]. The hydrazonoyl chlorides $\mathbf{2 a}-\mathbf{h}$ were synthesized according to procedures reported previously $[27,28]$.

$X$-ray diffraction data were collected on a Rigaku Oxford Diffraction Supernova diffractometer using $\mathrm{Cu} \mathrm{K} \alpha$ radiation, measured at a temperature of $120 \mathrm{~K}$ using a crystal of $5 \mathbf{b}$ immersed in cryo-oil and mounted in a loop. The CrysAlisPro [29] software package was used for cell refinement and data reduction. An analytical absorption correction (CrysAlisPro) was applied to the intensities before structure solution. The structure was solved by an intrinsic phasing method (SHELXT [30,31]). Structural refinement was carried out using the SHELXL [30] software with the SHELXLE [31] graphical user interface. Hydrogen atoms were positioned geometrically and constrained to ride on their parent atoms, with $\mathrm{C}-\mathrm{H}=0.95-1.00 \AA$ and $\mathrm{U}_{\text {iso }}=1.2-1.5 \cdot \mathrm{U}_{\text {eq }}$ (parent atom). The crystallographic details are summarized in Table S1.

\subsection{Synthesis of Cis- and Trans-[1,2,4]triazolo[4,3-a]quinazolin-5(3H)-one $\mathbf{4 a - g}$ and $\mathbf{5 a -} \mathbf{a}$}

A mixture of $0.5 \mathrm{mmol}$ of cyclohexane-fused 2-thioxopyrimidin-4-one 1 or 2 and $0.5 \mathrm{mmol}$ of hydrazonoyl chloride $(3 \mathbf{a}-\mathbf{g})$ in dioxane $(10 \mathrm{~mL})$ was treated at reflux temperature in the presence of $100 \mu \mathrm{L}$ of triethylamine (TEA) for $5-7 \mathrm{~h}$. The reactions were monitored by TLC ( $n$-hexane/EtOAC = 1:1 as the eluent) until completion. After solvent evaporation under reduced pressure, the residue was dissolved in $\mathrm{CHCl}_{3}(20 \mathrm{~mL})$, followed by extraction with water $(3 \times 10 \mathrm{~mL})$. The $\mathrm{CHCl}_{3}$ solution was dried on $\mathrm{Na}_{2} \mathrm{SO}_{4}$, the solvent was evaporated, and the residue was purified by column chromatography using $n$-hexane/EtOAC $=1: 1$ as the eluent.

$\left(5 a R^{*}, 9 a S^{*}\right)$-Ethyl 5-oxo-3-phenyl-3,5,5a,6,7,8,9,9a-octahydro-[1,2,4] triazolo[4,3-a]quinazoline-1carboxylate (4a): 69\%, m.p. $223-225{ }^{\circ} \mathrm{C}{ }^{1} \mathrm{H} \mathrm{NMR}\left(500 \mathrm{MHz}, \mathrm{CDCl}_{3}\right) \delta=8.09(\mathrm{~d}, J=7.7,2 \mathrm{H}), 7.45$ $(\mathrm{t}, J=8.0,2 \mathrm{H}), 7.33(\mathrm{t}, J=7.4,1 \mathrm{H}), 5.08-4.98(\mathrm{~m}, 1 \mathrm{H}, \mathrm{H}-4 \mathrm{a}), 4.58-4.45\left(\mathrm{~m}, 2 \mathrm{H}, \mathrm{CH}_{2} \mathrm{CH}_{3}\right), 2.92(\mathrm{~d}, J=4.2$, $1 \mathrm{H}), 2.68(\mathrm{~d}, J=12.5,1 \mathrm{H}), 2.03(\mathrm{~d}, J=9.5,1 \mathrm{H}), 1.86(\mathrm{~d}, J=10.9,1 \mathrm{H}), 1.47\left(\mathrm{t}, J=7.1,3 \mathrm{H}, \mathrm{CH}_{2} \mathrm{CH}_{3}\right)$, 1.51-1.41 (m, 5H). ${ }^{13} \mathrm{C} \mathrm{NMR}\left(126 \mathrm{MHz}, \mathrm{CDCl}_{3}\right) \delta=176.0(\mathrm{C}=\mathrm{O}), 156.3(\mathrm{C}=\mathrm{O}), 153.2(\mathrm{C}), 136.85(\mathrm{C})$, 136.3(C), 129.1(CH), 127.8(CH), 121.8(CH), 63.3( $\left.\mathrm{OCH}_{2}\right), 55.4(\mathrm{CH}), 55.2(\mathrm{CH}), 38.2\left(\mathrm{CH}_{2}\right), 28.8\left(\mathrm{CH}_{2}\right)$, 24.7 $\left(\mathrm{CH}_{2}\right), 24.6\left(\mathrm{CH}_{2}\right), 21.22(\mathrm{CH}), 14.29,14.1\left(\mathrm{CH}_{3}\right)$.

$\left(5 a R^{*}, 9 a S^{*}\right)$-Ethyl 5-oxo-3-(p-tolyl)-3,5,5a,6,7,8,9,9a-octahydro-[1,2,4]triazolo[4,3-a]quinazoline-1carboxylate (4b): $62 \%$, m.p. $263-264{ }^{\circ} \mathrm{C} .{ }^{1} \mathrm{H} \mathrm{NMR}\left(500 \mathrm{MHz}, \mathrm{CDCl}_{3}\right) \delta=7.94(\mathrm{~d}, J=8.5,2 \mathrm{H}), 7.24$ $(\mathrm{d}, J=8.3,2 \mathrm{H}), 5.10-4.95(\mathrm{~m}, 1 \mathrm{H}, \mathrm{H}-4 \mathrm{a}), 4.52\left(\mathrm{pd}, J=7.6,3.6,1 \mathrm{H}, \mathrm{CH}_{2} \mathrm{CH}_{3}\right), 2.91(\mathrm{~d}, J=5.5,1 \mathrm{H}$, H-8a), $2.68(\mathrm{~d}, J=12.2,1 \mathrm{H}), 2.37$ (s, 3H, p-tolyl), $2.03(\mathrm{~d}, J=12.1,1 \mathrm{H}), 1.86(\mathrm{~d}, J=11.1,1 \mathrm{H}), 1.47$ $\left(\mathrm{t}, J=7.1,3 \mathrm{H}, \mathrm{CH}_{2} \mathrm{CH}_{3}\right) .1 .62-1.4(\mathrm{~m}, 4 \mathrm{H}) .{ }^{13} \mathrm{C} \mathrm{NMR}\left(126 \mathrm{MHz} \mathrm{CDCl}_{3}\right) \delta=176.1(\mathrm{C}=\mathrm{O}), 156.4(\mathrm{C}=\mathrm{O})$, 153.1(C), 137.9(C), 136.6(C), 133.9(C), 129.7(CH), 121.7(CH), 63.3( $\left.\mathrm{OCH}_{2}\right)$, 55.3(CH), 38.1(CH), 28.8 ( $\left.\mathrm{CH}_{2}\right)$, 24.7 $\left(\mathrm{CH}_{2}\right), 24.6\left(\mathrm{CH}_{2}\right), 21.3\left(\mathrm{CH}_{2}\right), 21.11\left(\mathrm{CH}_{3}, p\right.$-tolyl $), 14.13\left(\mathrm{CH}_{2} \mathrm{CH}_{3}\right)$. 
(5aR*,9aS*)-Ethyl 5-oxo-3-(4-nitrophenyl)-3,5,5a,6,7,8,9,9a-octahydro-[1,2,4]triazolo[4,3-a]quinazoline-1carboxylate (4c): $61 \%$, m.p. $262-265{ }^{\circ} \mathrm{C} .{ }^{1} \mathrm{H} \mathrm{NMR}\left(500 \mathrm{MHz}, \mathrm{CDCl}_{3}\right) \delta=8.51(\mathrm{~d}, J=9.3,2 \mathrm{H}), 8.33$ $(\mathrm{d}, J=12.2,2 \mathrm{H}), 5.07(\mathrm{ddd}, J=11.3,6.5,4.4,1 \mathrm{H}), 4.60-4.49\left(\mathrm{~m}, 2 \mathrm{H}, \mathrm{CH}_{2} \mathrm{CH}_{3}\right), 2.95(\mathrm{~d}, J=6.0,1 \mathrm{H}), 2.68$ $(\mathrm{d}, J=8.0,1 \mathrm{H}), 2.05(\mathrm{~d}, J=12.5,1 \mathrm{H}), 1.88(\mathrm{~d}, J=10.2,1 \mathrm{H}), 1.50\left(\mathrm{t}, J=7.1,3 \mathrm{H}, \mathrm{CH}_{2} \mathrm{CH}_{3}\right), 1.63-1.43$ $(\mathrm{m}, 5 \mathrm{H}) .{ }^{13} \mathrm{C} \mathrm{NMR}\left(126 \mathrm{MHz}_{\mathrm{CDCl}}\right) \delta=176.0(\mathrm{C}=\mathrm{O}), 156.0(\mathrm{C}=\mathrm{O}), 155.6(\mathrm{C}), 146.0(\mathrm{C}), 141.4(\mathrm{C}), 137.6(\mathrm{C})$, 124.8(CH), 121.2(CH), 77.3( $\left.\mathrm{OCH}_{2}\right), 77.0(\mathrm{CH}), 76.8(\mathrm{CH}), 63.7\left(\mathrm{CH}_{2}\right)$, 55.5(CH), 38.2(CH), 28.8( $\left.\mathrm{CH}_{2}\right)$, 24.6( $\left(\mathrm{CH}_{2}\right), 24.5\left(\mathrm{CH}_{2}\right), 21.1\left(\mathrm{CH}_{2}\right), 14.11\left(\mathrm{CH}_{3}\right)$.

$\left(5 a R^{*}, 9 a S^{*}\right)$-Ethyl 5-oxo-3-(4-methoxyphenyl)-3,5,5a,6,7,8,9,9a-octahydro-[1,2,4]triazolo[4,3-a]quinazoline1-carboxylate (4d): 69\%, m.p. 215-216 ${ }^{\circ} \mathrm{C} .{ }^{1} \mathrm{H}$ NMR (500 MHz, $\left.\mathrm{CDCl}_{3}\right) \delta 7.94$ (d, J = 9.1 Hz, $\left.2 \mathrm{H}\right), 6.96$ $(\mathrm{d}, J=9.1 \mathrm{~Hz}, 2 \mathrm{H}), 5.15-4.92(\mathrm{~m}, 1 \mathrm{H}), 4.67-4.39\left(\mathrm{~m}, 2 \mathrm{H}, \mathrm{CH}_{2} \mathrm{CH}_{3}\right), 3.83(\mathrm{~s}, 3 \mathrm{H}), 2.93(\mathrm{~d}, J=5.7 \mathrm{~Hz}, 1 \mathrm{H})$, $2.68(\mathrm{~d}, J=12.2 \mathrm{~Hz}, 1 \mathrm{H}), 2.03(\mathrm{~d}, J=12.5 \mathrm{~Hz}, 1 \mathrm{H}), 1.86(\mathrm{~d}, J=12.5 \mathrm{~Hz}, 2 \mathrm{H}), 1.47(\mathrm{t}, J=7.1 \mathrm{~Hz}, 3 \mathrm{H}$, $\left.\mathrm{CH}_{2} \mathrm{CH}_{3}\right) .1 .62-1.4(\mathrm{~m}, 4 \mathrm{H}) .{ }^{13} \mathrm{C}$ NMR $\left(126 \mathrm{MHz}_{2} \mathrm{CDCl}_{3}\right) \delta 175.9(\mathrm{C}=\mathrm{O}), 159.1(\mathrm{C}=\mathrm{O}), 156.3(\mathrm{C}), 152.9(\mathrm{C})$, 136.6(C), 129.2(C), 123.7(CH), 114.3(CH), 63.3( $\left.\mathrm{OCH}_{2}\right), 55.6\left(\mathrm{OCH}_{3}\right), 55.4(\mathrm{CH}), 38.2(\mathrm{CH}), 28.8\left(\mathrm{CH}_{2}\right)$, $24.7\left(\mathrm{CH}_{2}\right), 24.62,2\left(\mathrm{CH}_{2}\right) \cdot 25,1\left(\mathrm{CH}_{2}\right) \cdot 14.1\left(\mathrm{CH}_{3}\right)$.

(5aR*,9aS*)-Ethyl 5-oxo-3-(4-chlorophenyl)-3,5,5a,6,7,8,9,9a-octahydro-[1,2,4]triazolo[4,3-a]quinazoline1-carboxylate (4e): $68 \%$, m.p. $239-241{ }^{\circ} \mathrm{C} .{ }^{1} \mathrm{H} \mathrm{NMR}\left(500 \mathrm{MHz}, \mathrm{CDCl}_{3}\right) \delta=8.12(\mathrm{~d}, J=9.0,2 \mathrm{H}), 7.42$ $(\mathrm{d}, J=9.1,2 \mathrm{H}), 5.04(\mathrm{ddd}, J=11.3,6.5,4.4,1 \mathrm{H}), 4.62-4.45\left(\mathrm{~m}, 2 \mathrm{H}, \mathrm{CH}_{2} \mathrm{CH}_{3}\right), 2.92(\mathrm{~d}, J=6.0,1 \mathrm{H}), 2.68$ $(\mathrm{d}, J=7.5,1 \mathrm{H}), 2.03(\mathrm{~d}, J=12.4,1 \mathrm{H}), 1.86(\mathrm{~d}, J=10.3,1 \mathrm{H}), 1.48\left(\mathrm{t}, J=7.1,3 \mathrm{H}, \mathrm{CH}_{2} \mathrm{CH}_{3}\right), 1.69-1.41$ $(\mathrm{m}, 5 \mathrm{H}) .{ }^{13} \mathrm{C}$ NMR $\left(126 \mathrm{MHz}_{\mathrm{CDCl}}\right) \delta=176.0(\mathrm{C}=\mathrm{O}), 156.2(\mathrm{C}=\mathrm{O}), 153.1(\mathrm{C}), 136.9(\mathrm{C}), 134.9(\mathrm{C}), 133.4(\mathrm{C})$, 129.3(CH), 122.7(CH), 63.5 $\left(\mathrm{OCH}_{2}\right), 55.4(\mathrm{CH}), 38.1(\mathrm{CH}), 28.8\left(\mathrm{CH}_{2}\right), 24.6\left(\mathrm{CH}_{2}\right), 24.6\left(\mathrm{CH}_{2}\right), 21.2\left(\mathrm{CH}_{2}\right)$, $14.1\left(\mathrm{CH}_{3}\right)$.

(5aR*,9aS*)-Ethyl 5-oxo-3-(4-(trifluoromethyl)phenyl)-3,5,5a,6,7,8,9,9a-octahydro-[1,2,4]triazolo[4,3-a] quinazoline-1-carboxylate (4f): $62 \%$, m.p. $202-206{ }^{\circ} \mathrm{C} .{ }^{1} \mathrm{H} \mathrm{NMR}\left(500 \mathrm{MHz}, \mathrm{CDCl}_{3}\right) \delta=8.56(\mathrm{dd}, J=8.3,3.0$, $1 \mathrm{H}), 8.27(\mathrm{~s}, 1 \mathrm{H}), 7.62-7.58(\mathrm{~m}, 2 \mathrm{H}), 5.20-4.89(\mathrm{~m}, 1 \mathrm{H}), 4.66-4.39\left(\mathrm{~m}, 2 \mathrm{H}, \mathrm{CH}_{2} \mathrm{CH}_{3}\right), 2.94(\mathrm{~d}, J=3.8,1 \mathrm{H})$, $2.68(\mathrm{~d}, J=9.9,1 \mathrm{H}), 2.05(\mathrm{dd}, J=8.7,3.7,1 \mathrm{H}), 1.87(\mathrm{~d}, J=10.2,1 \mathrm{H}), 1.74(\mathrm{~s}, 1 \mathrm{H}), 1.50(\mathrm{t}, J=7.1$, $\left.3 \mathrm{H}, \mathrm{CH}_{2} \mathrm{CH}_{3}\right), 1.61-1.41(\mathrm{~m}, 4 \mathrm{H}) .{ }^{13} \mathrm{C} \mathrm{NMR}\left(126 \mathrm{MHz}, \mathrm{CDCl}_{3}\right) \delta=176.02(\mathrm{C}=\mathrm{O}), 156.16(\mathrm{C}=\mathrm{O})$, 153.38(C), 137.2(C), 136.84(C), 131.7(q, $\left.J=38 \mathrm{~Hz}, \mathrm{CCF}_{3}\right), 129.9(\mathrm{CH}), 124.3\left(\mathrm{q}, J=3.5 \mathrm{~Hz}, \mathrm{CHCCF}_{3}\right)$, 123.5(q, $\left.J=273 \mathrm{~Hz}, \mathrm{CF}_{3}\right), 118.2\left(\mathrm{q}, \mathrm{J}=4 \mathrm{~Hz}, \mathrm{CHCCF}_{3}\right), 63.6\left(\mathrm{OCH}_{2}\right), 55.4(\mathrm{CH}), 38.2(\mathrm{CH}), 28.8\left(\mathrm{CH}_{2}\right)$, 24.6( $\left(\mathrm{CH}_{2}\right), 24.6\left(\mathrm{CH}_{2}\right), 21.2\left(\mathrm{CH}_{2}\right), 14.1\left(\mathrm{CH}_{3}\right)$.

$\left(5 a R^{*}, 9 a S^{*}\right)-1$-Acetyl-3-(p-tolyl)-5a,6,7,8,9,9a-hexahydro[1,2,4]triazolo[4,3-a]quinazoline-5(3H)-one (4g): 66\%, m.p. $196-198{ }^{\circ} \mathrm{C} .{ }^{1} \mathrm{H}$ NMR $\left(500 \mathrm{MHz} \mathrm{CDCl}_{3}\right) \delta=7.96(\mathrm{~d}, J=8.5,2 \mathrm{H}), 7.27(\mathrm{~d}, J=7.0,2 \mathrm{H}), 5.13-5.00$ $(\mathrm{m}, 1 \mathrm{H}), 2.92(\mathrm{~d}, J=2.3,1 \mathrm{H}), 2.69\left(\mathrm{~s}, 3 \mathrm{H}, \mathrm{COCH}_{3}\right), 2.66(\mathrm{~d}, J=7.7,1 \mathrm{H}), 2.39\left(\mathrm{~s}, 3 \mathrm{H}, \mathrm{CH}_{3}, p\right.$-tolyl), 1.98 $(\mathrm{d}, J=12.3,1 \mathrm{H}), 183(\mathrm{br}, 2 \mathrm{H}), 1.62-1.45(\mathrm{~m}, 4 \mathrm{H}) .{ }^{13} \mathrm{C} \mathrm{NMR}\left(126 \mathrm{MHz}, \mathrm{CDCl}_{3}\right) \delta 188.1(\mathrm{C}=\mathrm{O}), 176.0(\mathrm{C}=\mathrm{O})$, 153.6(C), 141.4(C), 138.1(C), 133.9(C), 129.8(CH), 121.6(CH), 55.0(CH), 38.2( $\left.\mathrm{CH}_{2}\right), 28.6\left(\mathrm{COCH}_{3}\right), 26.5$, 24.6( $\left(\mathrm{CH}_{2}\right), 24.5\left(\mathrm{CH}_{2}\right), 21.3\left(\mathrm{CH}_{2}\right), 21.1\left(\mathrm{CH}_{3}, p\right.$-tolyl $)$.

$\left(5 a R^{*}, 9 a R^{*}\right)$-Ethyl 5-oxo-3-phenyl-3,5,5a,6,7,8,9,9a-octahydro-[1,2,4]triazolo[4,3-a]quinazoline-1-carboxylate (5a): 65\%, m.p. 203-206 ${ }^{\circ} \mathrm{C} .{ }^{1} \mathrm{H}$ NMR $\left(500 \mathrm{MHz}, \mathrm{CDCl}_{3}\right) \delta 8.04(\mathrm{~d}, J=7.6 \mathrm{~Hz}, 2 \mathrm{H}), 7.48-7.41(\mathrm{~m}, 2 \mathrm{H})$, $7.33(\mathrm{t}, J=7.4 \mathrm{~Hz}, 1 \mathrm{H}), 4.58-4.44\left(\mathrm{~m}, 2 \mathrm{H}, \mathrm{CH}_{2} \mathrm{CH}_{3}\right), 4.08-3.97(\mathrm{~m}, 1 \mathrm{H}), 2.82(\mathrm{~d}, J=7.5 \mathrm{~Hz}, 1 \mathrm{H}), 2.50$ $(\mathrm{d}, J=13.0 \mathrm{~Hz}, 1 \mathrm{H}), 2.30-2.21(\mathrm{~m}, 2 \mathrm{H}), 1.94(\mathrm{t}, J=9.2 \mathrm{~Hz}, 1 \mathrm{H}), 1.46\left(\mathrm{t}, J=7.1 \mathrm{~Hz}, 3 \mathrm{H}, \mathrm{CH}_{2} \mathrm{CH}_{3}\right), 1.54-1.35$ (m. 4H). ${ }^{13} \mathrm{C} \mathrm{NMR}\left(126 \mathrm{MHz}, \mathrm{CDCl}_{3}\right) \delta 176.7(\mathrm{C}=\mathrm{O}), 157.5(\mathrm{C}=\mathrm{O}), 153.3(\mathrm{C}), 138.9(\mathrm{C}), 136.2(\mathrm{C}), 129.1(\mathrm{CH})$, 127.7(CH), 121.7(CH), 63.8( $\left(\mathrm{OCH}_{2}\right), 58.2(\mathrm{CH}), 43.4(\mathrm{CH}), 31.2\left(\mathrm{CH}_{2}\right), 25.4\left(\mathrm{CH}_{2}\right), 25.0\left(\mathrm{CH}_{2}\right), 24.2\left(\mathrm{CH}_{2}\right)$, 14.0. $\left(\mathrm{CH}_{3}\right)$.

$\left(5 a R^{*}, 9 a R^{*}\right)$-Ethyl5-oxo-3-(p-tolyl)-3,5,5a,6,7,8,9,9a-octahydro-[1,2,4]triazolo[4,3-a]quinazoline-1-carboxylate (5b): 67\%, m.p. $213-214{ }^{\circ} \mathrm{C}^{1} \mathrm{H}$ NMR $\left(500 \mathrm{MHz}, \mathrm{CDCl}_{3}\right) \delta 7.89(\mathrm{~d}, J=8.5 \mathrm{~Hz}, 2 \mathrm{H}), 7.24(\mathrm{~d}, J=8.2 \mathrm{~Hz}$, $2 \mathrm{H}), 4.71-4.35\left(\mathrm{~m}, 2 \mathrm{H}, \mathrm{CH}_{2} \mathrm{CH}_{3}\right), 4.17-3.87(\mathrm{~m}, 1 \mathrm{H}), 2.82(\mathrm{~d}, J=7.4 \mathrm{~Hz}, 1 \mathrm{H}), 2.50(\mathrm{~d}, J=10.5 \mathrm{~Hz}, 1 \mathrm{H})$, $2.37(\mathrm{~s}, 3 \mathrm{H}), 2.32-2.19(\mathrm{~m}, 1 \mathrm{H}), 1.93(\mathrm{t}, J=7.3 \mathrm{~Hz}, 2 \mathrm{H}), 1.46\left(\mathrm{t}, J=7.1 \mathrm{~Hz}, 3 \mathrm{H}, \mathrm{CH}_{2} \mathrm{CH}_{3}\right) .1 .47-1.132$ $(\mathrm{m}, 4 \mathrm{H}) .{ }^{13} \mathrm{C}$ NMR $\left(126 \mathrm{MHz}, \mathrm{CDCl}_{3}\right) \delta 176.7(\mathrm{C}=\mathrm{O}), 157.5(\mathrm{C}=\mathrm{O}), 153.2(\mathrm{C}), 138.7(\mathrm{C}), 137.8(\mathrm{C}), 133.8(\mathrm{C})$, 129.7(CH), 121.7(CH), 63.7 $\left(\mathrm{CH}_{2}\right), 58.2(\mathrm{CH}), 43.4(\mathrm{CH}), 31.2\left(\mathrm{CH}_{2}\right), 25.4\left(\mathrm{CH}_{2}\right), 25.1\left(\mathrm{CH}_{2}\right), 24.2\left(\mathrm{CH}_{2}\right)$, 21.1 $\left(\mathrm{CH}_{3}, p\right.$-tolyl), $14.0\left(\mathrm{CH}_{3}\right)$. 
(5aR*,9aR*)-Ethyl 5-oxo-3-(4-nitrophenyl)-3,5,5a,6,7,8,9,9a-octahydro-[1,2,4]triazolo[4,3-a]quinazoline-1carboxylate (5c): 71\%, m.p. $255-258{ }^{\circ} \mathrm{C} .{ }^{1} \mathrm{H} \mathrm{NMR}\left(500 \mathrm{MHz}, \mathrm{CDCl}_{3}\right) \delta 8.47-8.42(\mathrm{~m}, 2 \mathrm{H}), 8.34-8.27$ $(\mathrm{m}, 2 \mathrm{H}), 4.54\left(\mathrm{qq}, J=10.8,7.2 \mathrm{~Hz}, 2 \mathrm{H}, \mathrm{CH}_{2} \mathrm{CH}_{3}\right), 4.14-3.97(\mathrm{~m}, 1 \mathrm{H}), 2.90-2.70(\mathrm{~m}, 1 \mathrm{H}), 2.50(\mathrm{dd}, J=17.3$, $6.6 \mathrm{~Hz}, 1 \mathrm{H}), 2.33-2.20(\mathrm{~m}, 1 \mathrm{H}), 1.96(\mathrm{dd}, J=11.5,6.0 \mathrm{~Hz}, 2 \mathrm{H}), 1.49\left(\mathrm{t}, J=7.2 \mathrm{~Hz}, 3 \mathrm{H}, \mathrm{CH}_{2} \mathrm{CH}_{3}\right), 1.56-1.34$ $(\mathrm{m}, 4 \mathrm{H}) .{ }^{13} \mathrm{C}$ NMR $\left(126 \mathrm{MHz}, \mathrm{CDCl}_{3}\right) \delta 176.5(\mathrm{C}=\mathrm{O}), 157.2(\mathrm{C}=\mathrm{O}), 153.5(\mathrm{C}), 145.9(\mathrm{C}), 141.3(\mathrm{C}), 139.7(\mathrm{C})$, 124.8(CH), 121.0(CH), 64.1 $\left(\mathrm{OCH}_{2}\right), 58.2(\mathrm{CH}), 43.3(\mathrm{CH}), 31.1\left(\mathrm{CH}_{2}\right), 25.3\left(\mathrm{CH}_{2}\right), 24.9\left(\mathrm{CH}_{2}\right), 24.1\left(\mathrm{CH}_{2}\right)$, $14.0\left(\mathrm{CH}_{3}\right)$.

(5aR*,9aR*)-Ethyl 5-oxo-3-(4-methoxyphenyl)-3,5,5a,6,7,8,9,9a-octahydro-[1,2,4]triazolo[4,3-a]quinazoline1-carboxylate (5d): 69\%, m.p. 204-206 ${ }^{\circ} \mathrm{C}{ }^{1} \mathrm{H} \mathrm{NMR}\left(500 \mathrm{MHz}, \mathrm{CDCl}_{3}\right) \delta 7.92-7.85$ (m, 2H), 6.99-6.91 $(\mathrm{m}, 2 \mathrm{H}), 4.58-4.41\left(\mathrm{~m}, 2 \mathrm{H}, \mathrm{CH}_{2} \mathrm{CH}_{3}\right), 4.06-3.98(\mathrm{~m}, 1 \mathrm{H}), 3.83(\mathrm{~s}, 3 \mathrm{H}), 2.83(\mathrm{dt}, J=15.4,7.6 \mathrm{~Hz}, 1 \mathrm{H})$, $2.51(\mathrm{~d}, J=12.8 \mathrm{~Hz}, 1 \mathrm{H}), 2.28-2.20(\mathrm{~m}, 1 \mathrm{H}), 1.93(\mathrm{t}, J=7.9 \mathrm{~Hz}, 2 \mathrm{H}), 1.46\left(\mathrm{t}, J=7.1 \mathrm{~Hz}, 3 \mathrm{H}, \mathrm{CH}_{2} \mathrm{CH}_{3}\right)$, 1.53-1.25 (m,4H). ${ }^{13} \mathrm{C}$ NMR $\left(126 \mathrm{MHz}_{2} \mathrm{CDCl}_{3}\right) \delta 176.7(\mathrm{C}=\mathrm{O}), 159.0(\mathrm{C}=\mathrm{O}), 157.5(\mathrm{C}), 153.1(\mathrm{C}), 138.7(\mathrm{C})$, 129.3(C), 123.6(CH), 114.3(CH), 63.7( $\left.\mathrm{OCH}_{2}\right), 58.3(\mathrm{CH}), 55.6(\mathrm{CH}), 43.4\left(\mathrm{OCH}_{3}\right), 31.2\left(\mathrm{CH}_{2}\right), 25.4\left(\mathrm{CH}_{2}\right)$, $25.1\left(\mathrm{CH}_{2}\right), 24.2\left(\mathrm{CH}_{2}\right), 14.0\left(\mathrm{CH}_{3}\right)$.

(5aR*,9aR*)-Ethyl 5-oxo-3-(4-chlorophenyl)-3,5,5a,6,7,8,9,9a-octahydro-[1,2,4]triazolo[4,3-a]quinazoline1-carboxylate (5e): 68\%, m.p. 240-245 ${ }^{\circ} \mathrm{C} .{ }^{1} \mathrm{H} \mathrm{NMR}\left(500 \mathrm{MHz}, \mathrm{CDCl}_{3}\right) \delta 8.07$ (d, J = 8.9 Hz, $\left.2 \mathrm{H}\right), 7.41$ $(\mathrm{d}, J=8.9 \mathrm{~Hz}, 2 \mathrm{H}), 4.51\left(\mathrm{qq}, J=10.8,7.1 \mathrm{~Hz}, 2 \mathrm{H}, \mathrm{CH}_{2} \mathrm{CH}_{3}\right), 4.06-3.95(\mathrm{~m}, 1 \mathrm{H}), 2.79(\mathrm{~d}, J=7.7 \mathrm{~Hz}, 1 \mathrm{H})$, $2.49(\mathrm{~d}, J=12.5 \mathrm{~Hz}, 1 \mathrm{H}), 2.25(\mathrm{t}, J=12.2 \mathrm{~Hz}, 1 \mathrm{H}), 1.94(\mathrm{t}, J=8.7 \mathrm{~Hz}, 2 \mathrm{H}), 1.46\left(\mathrm{t}, J=7.1 \mathrm{~Hz}, 3 \mathrm{H}, \mathrm{CH}_{2} \mathrm{CH}_{3}\right)$, 1.53-1.26 (m, 4H). ${ }^{13} \mathrm{C} \mathrm{NMR}\left(126 \mathrm{MHz} \mathrm{CDCl}_{3}\right) \delta$ 176.4(C=O), 157.4(C=O), 153.2(C), 139.0(C), 134.9(C), 133.2(C), 129.2(CH), 122.6(CH), 63.8( $\left.\mathrm{OCH}_{2}\right), 58.3(\mathrm{CH}), 43.4(\mathrm{CH}), 31.2\left(\mathrm{CH}_{2}\right), 25.4\left(\mathrm{CH}_{2}\right), 25.0\left(\mathrm{CH}_{2}\right)$, 24.2( $\left(\mathrm{CH}_{2}\right), 14.0\left(\mathrm{CH}_{3}\right)$.

$\left(5 a R^{*}, 9 a R^{*}\right)$-Ethyl 5-oxo-3-(4-(trifluoromethyl)phenyl)-3,5,5a,6,7,8,9,9a-octahydro-[1,2,4]triazolo[4,3-a] quinazoline-1-carboxylate (5f): $70 \%$, m.p. $173-175^{\circ} \mathrm{C} .{ }^{1} \mathrm{H} \mathrm{NMR}\left(500 \mathrm{MHz}, \mathrm{CDCl}_{3}\right) \delta 8.60-8.42(\mathrm{~m}, 1 \mathrm{H}), 8.22$ $(\mathrm{d}, \mathrm{J}=0.6 \mathrm{~Hz}, 1 \mathrm{H}), 7.66-7.50(\mathrm{~m}, 2 \mathrm{H}), 4.60-4.36\left(\mathrm{~m}, 2 \mathrm{H}, \mathrm{CH}_{2} \mathrm{CH}_{3}\right), 4.13-3.93(\mathrm{~m}, 1 \mathrm{H}) .2 .80(\mathrm{~d}, J=8.0 \mathrm{~Hz}$, $1 \mathrm{H}), 2.50(\mathrm{~d}, J=13.0 \mathrm{~Hz}, 1 \mathrm{H}), 2.34-2.19(\mathrm{~m}, 1 \mathrm{H}), 1.95(\mathrm{t}, J=8.4 \mathrm{~Hz}, 2 \mathrm{H}), 1.48\left(\mathrm{t}, J=7.2 \mathrm{~Hz}, 3 \mathrm{H}, \mathrm{CH}_{2} \mathrm{CH}_{3}\right)$, 1.55-1.23 (m, 4H). ${ }^{13} \mathrm{C}$ NMR $\left(126 \mathrm{MHz} \mathrm{CDCl}_{3}\right) \delta 176.6(\mathrm{C}=\mathrm{O}), 157.3(\mathrm{C}=\mathrm{O}), 153.4(\mathrm{C}), 139.3(\mathrm{C}), 136.8(\mathrm{C})$, $131.7\left(\mathrm{q}, J=33 \mathrm{~Hz}, \mathrm{C}-\mathrm{CF}_{3}\right), 129.9,124.8,124.1\left(\mathrm{q}, J=3.6 \mathrm{~Hz}, \mathrm{CHCCF}_{3}\right), 123.4\left(\mathrm{q}, J=271 \mathrm{~Hz}, \mathrm{CF}_{3}\right), 118.1$ $(\mathrm{q}, \mathrm{J}=3.7 \mathrm{~Hz}), 64.0\left(\mathrm{OCH}_{2}\right), 58.2(\mathrm{CH}), 43.4(\mathrm{CH}), 31.2\left(\mathrm{CH}_{2}\right), 25.3\left(\mathrm{CH}_{2}\right), 25.0\left(\mathrm{CH}_{2}\right), 24.2\left(\mathrm{CH}_{2}\right), 14.0\left(\mathrm{CH}_{3}\right)$.

$\left(5 a R^{*}, 9 a R^{*}\right)-1-$ Acetyl-3-(p-tolyl)-5a,6,7,8,9,9a-hexahydro[1,2,4]triazolo[4,3-a]quinazoline-5(3H)-one (5g): 67\%, m.p. ${ }^{158-162 ~}{ }^{\circ} \mathrm{C}{ }^{1} \mathrm{H}$ NMR $\left(500 \mathrm{MHz} \mathrm{CDCl}_{3}\right) \delta 7.91(\mathrm{~d}, J=8.5 \mathrm{~Hz}, 2 \mathrm{H}), 7.26(\mathrm{~d}, J=8.2 \mathrm{~Hz}$, 2H), 4.06-3.98 (m, 1H), $2.92(\mathrm{~d}, J=8.5 \mathrm{~Hz}, 1 \mathrm{H}), 2.71(\mathrm{~s}, 3 \mathrm{H}), 2.49(\mathrm{~d}, J=13.2 \mathrm{~Hz}, 1 \mathrm{H}), 2.39$ (s, 3H), $2.25(\mathrm{t}, J=13.5 \mathrm{~Hz}, 1 \mathrm{H}), 1.92(\mathrm{~d}, J=11.0 \mathrm{~Hz}, 2 \mathrm{H}), 1.49(\mathrm{dd}, J=24.4,14.6 \mathrm{~Hz}, 1 \mathrm{H}), 1.42-1.22$ $(\mathrm{m}, 3 \mathrm{H}) .{ }^{13} \mathrm{C}$ NMR $\left(126 \mathrm{MHz}, \mathrm{CDCl}_{3}\right) \delta 187.9(\mathrm{C}=\mathrm{O}), 176.8(\mathrm{C}=\mathrm{O}), 153.7(\mathrm{C}), 144.2(\mathrm{C}), 138.0(\mathrm{C}), 133.8(\mathrm{C})$, 129.7(CH), 121.6(CH), 58.5( $\left.\mathrm{COCH}_{3}\right), 43.6(\mathrm{CH}), 31.9\left(\mathrm{CH}_{2}\right), 27.6(\mathrm{CH}), 25.5\left(\mathrm{CH}_{2}\right), 25.2\left(\mathrm{CH}_{2}\right), 24.3\left(\mathrm{CH}_{2}\right)$, 21.1( $\mathrm{CH}_{3}, p$-tolyl).

\section{Conclusions}

Herein, we report the unexpected regioselectivity of the reaction between 2-thioxopyrimidin-4-ones with hydrazonoyl chlorides to produce the angular regioisomers [1,2,4]triazolo[4,3-a]quinazolin-5-ones, rather than the linear isomers [1,2,4] triazolo[4,3-a]quinazolin-5-ones. The transformations are controlled by electronic factors of 2-thioxopyrimidin-4-one. This phenomenon was exploited in the synthesis of the novel stereoisomeric octahydro[1,2,4]triazolo[4,3-a]quinazolin-5-ones $\mathbf{4} \mathbf{a}-\mathbf{g}$ and $\mathbf{5 a} \mathbf{a} \mathbf{g}$ starting from cis or trans cyclohexane-fused 2-thioxopyrimidin-4-one $\mathbf{1}$ or $\mathbf{2}$, respectively. The stereochemistry of the products was assigned on the basis of one- and two-dimensional NMR spectra and by X-ray measurements providing conclusive evidence.

Supplementary Materials: NMR spectra of all the synthesized compounds and crystallographic data for $5 \mathbf{b}$ are available online.

Author Contributions: F.F., A.I.S., and M.P. planned and designed the project. A.I.S. and M.P. performed the syntheses and characterized the synthesized compounds. M.H. performed and analyzed the X-ray measurements of compound $5 \mathrm{~b}$. A.I.S. prepared the manuscript for publication, and all the authors discussed 
the results and commented on the manuscript. All authors have read and agreed to the published version of the manuscript.

Funding: We are grateful to the Hungarian Research Foundation (OTKA No. K 115731). The financial support of the GINOP-2.3.2-15-2016-00014 project is acknowledged. The Ministry of Human Capacities, Hungary, grant TUDFO/47138-1/2019, is acknowledged.

Conflicts of Interest: The authors declare no conflict of interest.

\section{References}

1. Fan, W.-Q.; Katritzky, A.R. 1,2,3-Triazoles. In Comprehensive Heterocycle Chemistry II; Katritzky, A.R., Rees, C.W., Scriven, E.F.V., Eds.; Pergamon Press: New York, NY, USA, 1996; Volume 4, pp. 1-126.

2. Su, N.N.; Li, Y.; Yu, S.J.; Zhang, X.; Liu, X.H.; Zhao, W.G. Microwave-assisted synthesis of some novel 1,2,3-triazoles by click chemistry, and their biological activity. Res. Chem. Intermed. 2013, 39, 759-766. [CrossRef]

3. Astakhov, A.V.; Chernyshev, V.M. Molecular structure of 3-amino[1,2,4]triazolo-[4,3-a] pyrimidin-5-one in various tautomeric forms: Investigation by DFT and QTAIM methods. Chem. Heterocycl. Compd. 2014, 50, 319-326. [CrossRef]

4. Abdelhamid, A.O.; Gomha, S.M.; Abdelriheem, N.A.; Kandeel, S.M. Synthesis of New 3-Heteroarylindoles as Potential Anticancer Agents. Molecules 2016, 21, 929. [CrossRef]

5. Gomha, S.M. A facile one-pot synthesis of 6,7,8,9-tetrahydrobenzo[4,5]thieno[2,3- $d]-1,2,4$-triazolo [4,5-a]pyrimidin-5-ones. Mon. Chem. 2009, 140, 213-220. [CrossRef]

6. Fares, M.; Abou-Seri, S.M.; Abdel-Aziz, H.A.; Abbas, S.E.S.; Youssef, M.M.; Eladwy, R.A. Synthesis and antitumor activity of pyrido[2,3- $d$ ] pyrimidine and pyrido[2,3- $d][1,2,4]$ triazolo[4,3-a]pyrimidine derivatives that induce apoptosis through G(1) cell-cycle arrest. Eur. J. Med. Chem. 2014, 83, 155-166. [CrossRef]

7. Gomha, S.M.; Ahmed, S.A.; Abdelhamid, A.O. Synthesis and cytotoxicity evaluation of some novel thiazoles, thiadiazoles, and pyrido[2,3- $d][1,2,4]$ triazolo[4,3-a]pyrimidin-5(1H)-one incorporating triazole moiety. Molecules 2015, 20, 1357-1376. [CrossRef]

8. Liu, X.H.; Sun, Z.H.; Yang, M.Y.; Tan, C.X.; Weng, J.Q.; Zhang, Y.G.; Ma, Y. Microwave assistant one pot synthesis, crystal structure, antifungal activities and 3D-QSAR of novel 1,2,4-triazolo[4,3-a]pyridines. Chem. Biol. Drug Des. 2014, 84, 342-347. [CrossRef]

9. Gomha, S.M.; Badrey, M.G. Ecofriendly regioselective one-pot synthesis of chromeno[4,3- $d][1,2,4]$ triazolo [4,3-a]pyrimidine. Eur. J. Chem. 2013, 4, 180-184. [CrossRef]

10. Dieckmann, W.; Platz, L. Ueber eine neue Bildungsweise von Osotetrazonen. Ber. Dtsch. Chem. Ges. 1905, 38, 2986-2990. [CrossRef]

11. Silvestri, R.; Cascio, M.G.; La Regina, G.; Piscitelli, F.; Lavecchia, A.; Brizzi, A.; Pasquini, S.; Botta, M.; Novellino, E.; Di Marzo, V.; et al. Synthesis, cannabinoid receptor affinity, and molecular modeling studies of substituted 1-aryl-5-(1H-pyrrol-1-yl)-1H-pyrazole-3-carboxamides. J. Med. Chem. 2008, 51, 1560-1576. [CrossRef]

12. Liu, J.; Nie, M.; Wang, Y.; Hu, J.; Zhang, F.; Gao, Y.; Liu, Y.; Gong, P. Design, synthesis and structure-activity relationships of novel 4-phenoxyquinoline derivatives containing 1,2,4-triazolone moiety as c-Met kinase inhibitors. Eur. J. Med. Chem. 2016, 123, 431-446. [CrossRef]

13. Abdelhamid, A.O.; Shawali, A.S.; Gomha, S.M.; El-Enany, W.M.A. Synthesis and antimicrobial evaluation of some novel thiazole, 1,3,4-thiadiazole and pyrido[2,3-d][1,2,4]triazolo[4,3-a]pyrimidine derivatives incorporating pyrazole moiety. Heterocycles 2015, 91, 2126-2142. [CrossRef]

14. Riyadh, S.M. Enaminones as building blocks for the synthesis of substituted pyrazoles with antitumor and antimicrobial activities. Molecules 2011, 16, 1834-1853. [CrossRef]

15. Said, A.I.; Palkó, M.; Haukka, M.; Fülöp, F. Retro Diels Alder Protocol for Regioselective Synthesis of Novel [1,2,4]triazolo[4,3-a]pyrimidin-7(1H)-ones. RSC Adv. 2020, 10, 33937-33943. [CrossRef]

16. Said, A.I.; Haukka, M.; Fülöp, F. Microwave-Assisted Regioselective Synthesis of Variously Functionalized [1,2,4]triazolo[3,4-b]quinazolin-5(1H)-ones. Curr. Org. Chem. 2020, 24, 1892-1896. [CrossRef]

17. Hassaneen, H.M.; Abdelhadi, H.A.; Abdallah, T.A. Novel synthesis of 1,2,4-triazolo[4,3-a]pyrimidin-5-one derivatives. Tetrahedron 2001, 57, 10133-10138. [CrossRef] 
18. Hassneen, H.M.; Abdallah, T.A. New Routes to Pyridino[2,3- $d$ ]pyrimidin-4-one and Pyridino[2,3- $d]$ triazolino[4,5-a]pyrimidin-5-one Derivatives. Molecules 2003, 8, 333-341. [CrossRef]

19. Abdel Hafez, N.A.; Farghaly, T.A.; Al-Omar, M.A.; Abdall, M.M. Synthesis of bioactive polyheterocyclic ring systems as $5 \alpha$-reductase inhibitors. Eur. J. Med. Chem. 2010, 45, 4838-4844. [CrossRef]

20. Abdallah, M.A.; Gomha, S.M.; Morad, M.A.; Elaasser, M.M. Synthesis of Pyridotriazolopyrimidines as Antitumor Agents. J. Heterocyclic Chem. 2017, 54, 1242-1251. [CrossRef]

21. Abdallah, T.A.; Darwish, M.A.; Hassaneen, H.M. A Novel Synthesis of 1,2,4-Triazolopteridines. Molecules 2002, 7, 494-500. [CrossRef]

22. Sohár, P.; Szöke-Molnár, Z.; Stájer, G.; Bernáth, G. Preparation and structure of cycloalkane-condensed [1,3]thiazino[3,2-a] pyrimidinones. Magn. Reson. Chem. 1989, 27, 959-963. [CrossRef]

23. Elliott, A.J.; Callaghan, P.D.; Gibson, M.S.; Nemeth, S.T. Rearrangement of arylthiohydrazonates. Can. J. Chem. 1975, 53, 1484-1490. [CrossRef]

24. Shawali, A.S.; Abbas, I.M.; Mahran, A.M. Facile Entries for Regioselective Synthesis of $[1,2,4]$ Triazolo [4,3-a]pyrimidin-5(1H)-ones from 2-Thiouracil. JICS 2004, 1, 33-39. [CrossRef]

25. Stajer, G.; Szabo, A.E.; Sohar, P. Synthesis and structure of norbornane/ene-fused thiouracils and thiazino[3,2-a]pyrimidinones. Heterocycles 1999, 51, 1849-1854. [CrossRef]

26. Stájer, G.; Szabó, A.E.; Pintye, J.; Bernáth, G.; Sohár, P. Stereochemical Studies. Part 86. Saturated Heterocycles. Part 81 Preparation of New Thiouracils via Retrodiene Decomposition of Methylene-bridged Quinazolone Thiones. J. Chem. Soc. Perkin Trans. I 1985, 2483-2487. [CrossRef]

27. Soliman, H.M.; Basuny, A.M.; Arafat, S.M. Utilization of Stearic acid Extracted from Olive Pomace for Production of Triazoles, Thiadiazoles and Thiadiazines Derivatives of Potential Biological Activities. J. Oleo. Sci. 2015, 64, 1019-1032. [CrossRef]

28. Matiychuk, V.S.; Potopnyk, M.A.; Luboradzki, R.; Obushak, M.D. New Method for the Synthesis of 1-Aryl-1,2,4-triazole Derivatives. Synthesis 2011, 11, 1799-1813. [CrossRef]

29. Rikagu Oxford Diffraction. CrysAlisPro; Agilent Technologies Inc.: Yarnton, UK, 2018.

30. Sheldrick, G.M. Crystal structure refinement with SHELXL. Acta Cryst. 2015, C71, 3-8. [CrossRef]

31. Hübschle, C.B.; Sheldrick, G.M.; Dittrich, B. ShelXle: A Qt graphical user interface for SHELXL. J. Appl. Cryst. 2011, 44, 1281-1284. [CrossRef]

Sample Availability: Samples of all compounds are available from the authors.

Publisher's Note: MDPI stays neutral with regard to jurisdictional claims in published maps and institutional affiliations.

(C) 2020 by the authors. Licensee MDPI, Basel, Switzerland. This article is an open access article distributed under the terms and conditions of the Creative Commons Attribution (CC BY) license (http://creativecommons.org/licenses/by/4.0/). 